EPJ Web of Conferences 59, 01006 (2013)

DOI: $10.1051 /$ epjconf/20135901006

(C) Owned by the authors, published by EDP Sciences, 2013

\title{
HiPER: The European path to laser energy
}

\section{Chris Edwards ${ }^{1}$ and François Amiranoff ${ }^{2}$ on behalf of the HiPER Consortium}

\author{
1 Central Laser Facility, S.T.F.C. Rutherford Appleton Laboratory, Didcot OX11 0QX, UK \\ 2 LULI, Ecole Polytechnique, CNRS, CEA, UPMC, 91128 Palaiseau, France
}

\begin{abstract}
While for decades, energy production relying on laser inertial fusion has been a strong motivation for the development in Europe of a few high-energy laser facilities and dedicated scientific programs, the HiPER initiative launched in 2004 fostered an ambitious large-scale coordinated European program toward inertial fusion energy. Anticipating the successful demonstration of fusion ignition and gain at the National Ignition Facility (NIF) in the USA, scientists and engineers from across Europe are developing the case for a next generation laser fusion facility, HiPER, to be constructed in Europe. The single-facility build strategy of HiPER (High Power Laser Energy Research Facility) aims at first demonstrating some key elements of a fusion reactor in a high rep-rate few-second cycle mode, before addressing energy production on a high rep-rate continuous mode in a second area.
\end{abstract}

It has been known for almost hundred years that stars produce their energy by fusion of light elements, starting from fusion of hydrogen nuclei into helium. However, the conditions in which such a fusion process can be used to produce energy on a large scale in a controlled manner are so difficult to reach on Earth that a fusion-based power plant is still to be designed. The easiest fusion reaction is that of D and $\mathrm{T}$ producing a ${ }^{4} \mathrm{He}$ nucleus and a neutron with respective energies of $3.5 \mathrm{MeV}$ and $14.1 \mathrm{MeV}$. In this case, in addition to an almost unlimited quantity of available D atoms, the radioactive waste generated from a fusion energy plant due to neutron activation of the fusion chamber will be short-lived and can be recycled within a 100-year period with appropriate choice of existing materials. Moreover, a fusion energy plant produces no carbon during the electricity generation cycle, even if there will be a carbon footprint associated with the construction of plant and delivery of materials. If successfully developed, fusion energy may thus provide a sustainable, low carbon, environmentally acceptable, safe and secure commercial energy source without the long-lived radioactive waste associated with conventional nuclear power plants.

In the case of laser inertial fusion, a mg-mass of D-T fuel is enclosed inside a mm-size microsphere directly irradiated by powerful laser beams (direct drive) or by x-rays generated inside a laser-irradiated high- $Z$ cavity (indirect drive). The expansion of the heated outer part of the microsphere generates a compression of the inner part of this shell and of the fuel. If sufficiently compressed, the heating of the core of the fuel leads to the conditions necessary for the start of fusion reactions and possibly to the ignition of a large fraction of the fuel.

After more than fifty years of fruitful research programs and development of dedicated laser and experimental facilities, it is now believed that a laser energy close to 1 to $2 \mathrm{MJ}$ should be sufficient to lead to fuel ignition and energy gains between the input laser energy and the energy produced by fusion reactions of the order or larger than 10. The demonstration of high gains in the indirect-drive scheme is expected in the next few months or years on the NIF (National Ignition Facility) in the USA and on the LMJ (Laser MegaJoule) in France, two facilities at the MJ level.

This is an Open Access article distributed under the terms of the Creative Commons Attribution License 2.0, which permits unrestricted use, distribution, and reproduction in any medium, provided the original work is properly cited. 


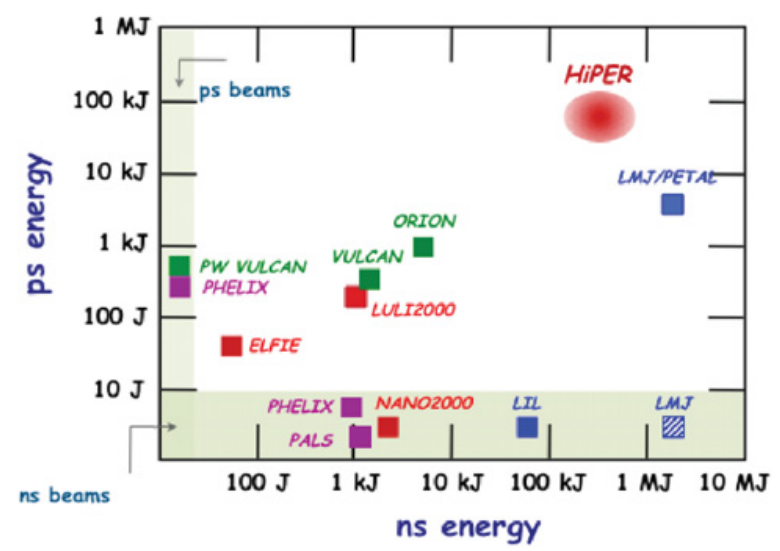

Figure 1. Large-scale European laser facilities and their parameters in the ns and ps regimes.

The route is but very long from a proof-of-principle demonstration of gain to a viable power plant based on laser fusion, and success will require the complementary expertise of scientists and engineers in various fields. The objective of the European consortium HiPER [1, 2] is precisely to benefit from the European expertise to pave the way to future power plants based on laser fusion. In addition to the intrinsic political and financial issues of such a challenge, the HiPER strategy thus includes addressing the numerous scientific and technological themes relevant to a reactor.

The first step of the HiPER program, and a main element in the frame of the preparatory phase ending in April 2013, is to prepare a business case for laser-based energy production. Addressing such points as the possible contribution of laser energy in a global energy portfolio, economical evaluations as well as scientific and technological bottlenecks to be addressed, this business case will draw the route for the preparation of a detailed proposal for the construction of a dedicated laser facility. This 7 to 10 years long program will include the choice of the best ignition scheme, the design of all the elements to be included in the facility and the development of specific prototypes including a $10 \mathrm{~kJ}$, $10 \mathrm{~Hz}$ diode-pumped laser chain.

Extensive financial modelling has been carried out during the HiPER Preparatory Phase. The results show that to be commercially attractive, the fusion cycle must run at a repetition rate of at least 10 cycles per second, and that a "target gain" (laser energy input divided by fusion energy output) in the region of 60-70 is required. The models also identify key technical and economic performance criteria; the efficiency of the lasers, for example, and the maximum cost of the targets, for commercial viability.

The demonstration of ignition will most probably be obtained in the indirect-drive configuration. However, because of the connections of such a scheme with defence programs, HiPER will concentrate on direct-drive schemes, also expected to be more efficient. From the beginning, HiPER strategy has been to try and validate alternative schemes less demanding in terms of laser energy, and thus hopefully leading to less expensive facilities. Fast ignition and shock ignition are presently the preferred schemes. A large effort is being made toward shock ignition, a supposedly simpler scheme (at least laser-wise because it does not require short-pulse ps high-power beams) that could be tested on NIF and LMJ without large additional investments.

To complement the shock ignition activity, UK will also study indirect drive schemes in collaboration with academia and Lawrence Livermore National Laboratory in the U.S. While this work will be conducted outside of the HiPER project, results will be reported regularly to HiPER.

To address the physics of laser inertial fusion, and more generally the physics of laser-matter interaction, laser-generated plasmas and applications, a set of European large-scale facilities offer access to the scientific community (cf. Fig. 1). These include VULCAN and PW VULCAN in the CLF and 


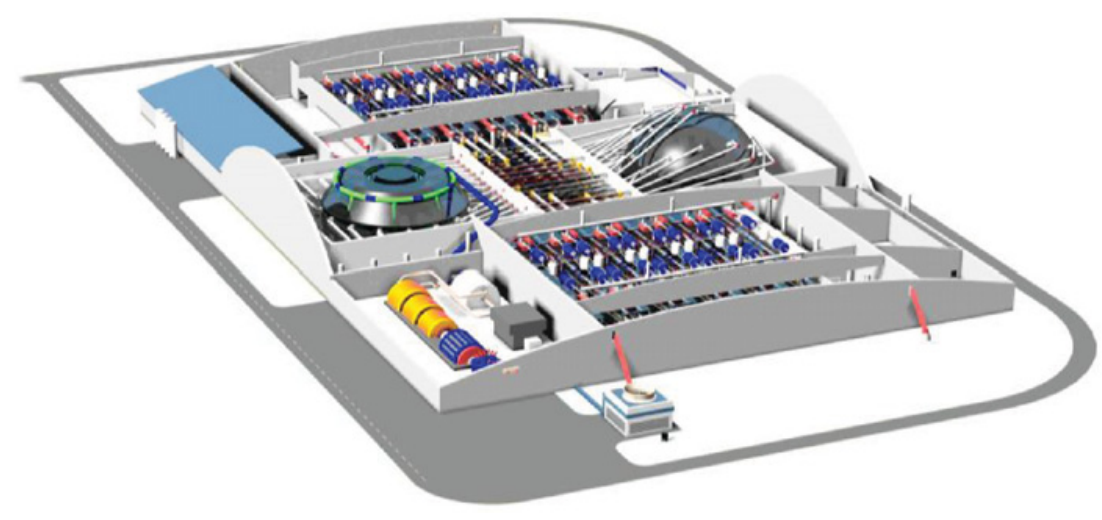

Figure 2. Artist view of the HiPER facility including laser driver and 2 experimental areas.

ORION in AWE in the UK, ELFIE and LULI2000 in LULI and LIL in CEA/CESTA in France, PHELIX in GSI in Germany and PALS in Prague in Czech Republic. Most of these facilities couple high-energy long and short laser pulses. In CEA/CESTA in Aquitaine, LMJ and PETAL, a multi-PW ps beam, will be available from about 2015. The HiPER symbol corresponds to the estimation of the energies required for the fast-ignition scheme. In case shock-ignition is chosen, HiPER would not require ps beams (except for diagnostic purposes).

In addition to these European facilities, it is expected that a significant part of the program will be developed on large facilities in the USA (OMEGA and NIF) and in Japan (LFEX).

In parallel to the studies on laser fusion physics and demonstration of ignition, a major challenge is the development of reactor designs and associated technologies. The principle of a laser-based fusion reactor is the following. Ten times per second, a pellet containing the D-T fuel is injected in the middle of a large vacuum chamber where it is irradiated by tens to hundreds of laser beams. Fusion takes place and a large energy, up to tens of $\mathrm{MJ}$, is released in $14.1 \mathrm{MeV}$ neutrons. These escaping neutrons are captured in a blanket surrounding the vacuum chamber and containing $\mathrm{Li}$.

The blanket is heated and interaction with $\mathrm{Li}$ generates additional Tritium. The process heat is then used to drive conventional, high-efficiency electricity generating plant. These apparently simple sentences hide tremendously difficult challenges among which high-energy high-rep-rate laser drivers, large optics, target mass-production, injection, tracking and positioning, chamber design, material resistance, material activation, radiation issues, remote handling, and many others. The HiPER community already addresses many of these themes [3, and papers in this issue].

A first design of the HiPER facility is shown in Fig. 2.

The following time schedule summarises the next steps of the HiPER program. As explained before, the next 7 to 9 years will be dedicated to technological developments, risk reduction and preparation of a technical design report for the HiPER facility. In order to reduce the time from the first tests to the demonstration of production of electricity, it has been decided to design a single facility to address two successive steps. In a first target area, scale-1 tests will be pursued with high gain targets on specific topics such as high-rep-rate laser driver, target injection and tracking, laser beam alignment and more generally the design of the chamber. In this first part of the program the facility will be operated in burst mode, typically at $10 \mathrm{~Hz}$ during $10 \mathrm{~s}$ with a total of 5 high-gain D-T shots per burst. In a second step, a dedicated target area will be designed to run in a high-gain continuous mode with 10 high-gain targets per second. It will address all the themes relevant to a reactor, including blanket, tritium handling, chamber material and production of electricity. This time-schedule is most probably optimistic. It assumes that money, people will be available at the right level at the right time, and that industry will be able to deliver the components of the facility. 


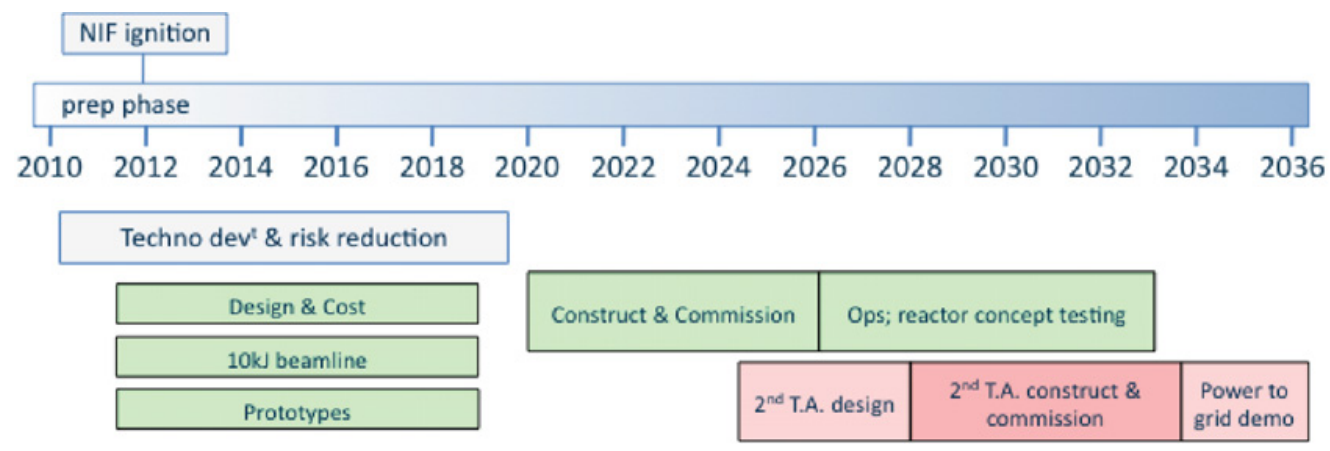

Figure 3. Time-schedule of the HiPER program.

However, the system separability will be a major advantage to accelerate delivery. The laser driver is located remotely from the fusion chamber, with the laser beam energy relayed by optics to the pellet interaction point. This is advantageous for a number of reasons. First, the laser, a high value capital asset, is not subjected to the neutron flux and is therefore isolated from this potential source of damage. Second, by incorporating redundancy into the laser driver, which consists of a large number of identical beamlines, maintenance can be carried out "live", without interrupting power generation. This increases the availability of the system for operations and simplifies maintenance, two crucial requirements of utility operators. Third, the driver design and performance can be the subject of further improvements, demonstrated on a single prototype beamline, and then implemented via a rolling installation programme in operating plants. In the context of the single facility pilot plant strategy, incorporation of new laser drivers does not require the construction of a new facility. In the same way, new blanket designs, improved target manufacturing techniques and new first wall materials can be incorporated within the existing facility without the need for major shut-downs.

In conclusion, the way to laser energy, the production of electricity using laser inertial fusion, is still long and uncertain. But, after 50 years of fruitful research and technological advances, the expected demonstration of high-gain ignition will be a major scientific, technological as well as political step. Thanks to the HiPER project, a large European consortium now concentrates on the objective of laser energy. The European expertise on plasma physics, high-power lasers and all technologies relevant to a fusion reactor is already established. It will be the task for the years to come to strengthen the links between a large number of actors in collaboration with USA and Japan, toward a very challenging but most promising goal.

In addition to national funding allocated to individual partners, the HiPER program is supported by the European Community (FP7 project number 211737), the Science and Technology Council of the United Kingdom, and MŠMT, the Ministry of Education, Youth and Sports of the Czech Republic.

\section{References}

[1] http://www.hiper-laser.org/index.asp

[2] M. Dunne, Nature Physics 2, 2 (2006)

[3] Diode-Pumped High Energy and High Power Lasers; ELI: Ultrarelativistic Laser-Matter Interactions and Petawatt Photonics; and HiPER: the European Pathway to Laser Energy, edited by J. Hein, L. O. Silva, G. Korn, L. A. Gizzi, C. Edwards, Proc. of SPIE Vol. 8080, 80802M 